\title{
两种对流参数化方案下降水和潜热加热空间 结构的模拟及其影响
}

\author{
王晓聪 ${ }^{(1)(3)}$ ，包庆 ${ }^{(1)}$ ，刘琨 ${ }^{(3)}$ ，吴国雄 ${ }^{(1)}$ ，刘屹岷 ${ }^{(1)}$ \\ (1) 中国科学院大气物理研究所大气科学和地球流体力学数值模拟国家重点实验室, 北京 100029 ; \\ (2) 中国科学院研究生院, 北京 100049; \\ (3) 中国气象局数值预报中心, 北京 100081 \\ * 联系人, E-mail: lym@lasg.iap.ac.cn
}

收稿日期: 2011-04-06; 接受日期: 2011-11-08

中国科学院战略性先异科技专项(编号: XDA05110303)和国家自然科学基金(批准号: 40925015, 40875034, 41023002)资助

摘要利用中国科学院大气物理研究所大气科学和地球流体力学数值模拟国家重点实验室 (LASG/IAP)新版大气环流模式 SAMIL(Spectral Atmosphere Model of IAP LASG), 分析比较了 Manabe 和 Tiedtke 两种对流方案对降水和潜热加热空间结构的模拟性能. 结果表明, Tiedtke 方 案模拟的对流降水偏强, 层状降水偏弱, 致使层状降水占总降水比例低于观测. 与此相比, Manabe 方案较为合理地再现了沿赤道的层状降水中心, 但其模拟的孟加拉湾降水中心向东向 北伸展, 在高原下游有虚假强降水出现. 通过比较两种对流方案模拟的潜热加热空间结构, 结 果表明 Tiedtke 方案的模拟结果远较 Manabe 方案合理, 表现为与观测一致的位于对流层中层的 对流加热峰值, 以及 $0^{\circ} \mathrm{C}$ 层之上的层状加热与其下的冷却. 而 Manabe 方案模拟的对流加热峰值 所在高度较观测偏低, 大致位于 $700 \mathrm{hPa}$. 另外对于层状加热, Manabe 方案模拟不出 $0^{\circ} \mathrm{C}$ 层以下 的冷却, 而表现为整层加热. 温度、比湿等要素的 Taylor 图表明: Tiedtke 方案的模拟结果与 ERA40, NCEP 再分析资料的相关系数更高, 尤其是对流层高层 $200 \mathrm{hPa}$ 温度的模拟. 文中同时 提出 Tiedtke 方案下层状降水模拟失真的可能原因是层状降水方案忽略了积云对流过程中卷出 水凝物的影响, 该猜想通过敏感性试验得到证实.

\section{关键词}

Manabe 方案

Tiedtke 方案

层状降水

对流降水

潜热加热

SAMIL
实际大气中, 积云对流与大尺度大气环流的相 互作用对大气环流与气候变化有着重要影响. 积云 对流不仅可以产生强烈的降水及云辐射强迫, 而且 其深厚的垂直运动很大程度上影响着大气中温度、水 汽的垂直分布以及经向和纬向环流, 是全球能量、水 分和质量循环的重要调节器. 如何在数值天气模式
以及气候模式中合理地描述积云对流一直是国内外 研究的热点, 由于积云尺度小于模式网格尺度, 描述 这一次网格物理过程的方法通常是参数化. 自 Smagorinsky ${ }^{[1]}$ 首次将对流参数化方法一一湿绝热过 程引入到数值模式中以来, 先后提出了多种积云对 流参数化方案, 概括起来主要有三种: (1) Manabe 对

英文引用格式: Wang X C, Bao Q, Liu K, et al. Features of rainfall and latent heating structure simulated by two convective parameterization schemes. Sci China Earth Sci, 2011, 54: 1779-1788, doi: 10.1007/s11430-011-4282-2 
流调整型方案 ${ }^{[2]}$ (简写为 Manabe 方案); (2) 基于水汽 辐合、辐散的 Kuo 参数化方案 ${ }^{[3]}$; (3) 考虑积云与环 境大气相互作用的 Arakawa-Schubert 方案 ${ }^{[4]}$, Tiedtke ${ }^{[5]}$ 在此基础上发展了采用总体云模型的质量 通量积云参数化方案, 并成功应用于欧洲中期数值 预报(ECMWF)(简写为 Tiedtke 方案).

鉴于积云对流过程在模式中的重要性, 关于不 同积云对流方案的评估国内外学者做过很多研究， 但更多地是针对中尺度模式或数值天气模式比较不 同积云对流参数化方案对某一天气过程的模拟 ${ }^{[6]}$. 气 候模式中积云对流参数化方案的评估一方面通过比 较模拟的降水气候态以及环流场 ${ }^{[7,8]}$, 如 Liu 等 ${ }^{[9]}$ 通过 水球试验研究了不同积云对流方案对赤道辐合带 (ITCZ)模拟的敏感性. 另一方面, ENSO 和 MJO 的模 拟能力也是反映对流参数化方案优劣的一个重要指 标 ${ }^{[10,11]}$. 此外, 积云对流还可以通过影响大气辐射过 程, 改变辐射通量, 从而对局地能量收支产生影响 ${ }^{[12]}$. 质量通量型对流参数化方案还具有积云对物质的垂直 输送功能, 其模拟能力也能反映参数化方案的优劣 ${ }^{[13]}$.

最近, Lin 等 ${ }^{[14]}$ 研究表明层状凝结过程对大气低 频振荡(MJO)同样具有重要作用, MJO 湿位相期, 总 降水异常的 $60 \%$ 都来自层状降水, 由层状降水引起 的凝结加热表现为冻结层 $\left(0^{\circ} \mathrm{C}\right.$ 层 $)$ 之上的加热与其下 的冷却, 对流凝结加热表现为整层加热, 总凝结加热 在垂直剖面上呈“top-heavy”结构(对流层高层强加热, 低层弱加热). 模式对 MJO 模拟的好坏, 某种程度上 取决于对流降水与层状降水的模拟.

本文基于中国科学院大气物理研究所大气科学 和地球流体力学数值模拟国家重点实验室 (LASG/IAP) 新版大气环流模式 SAMIL, 利用 TRMM 卫星资料, 分析比较了 Manabe 和 Tiedtke 两种对流方 案模拟的对流降水及层状降水, 并揭示其对应的潜 热加热空间结构; 利用 ERA40 和 NCEP 再分析资料 比较了两种对流方案对环流场的模拟能力, 旨在发 现模式的不足以及可能解决途径, 以期进一步改进 发展模式, 提高模式对降水、潜热加热及环流场的模 拟能力, 进而改善模式的整体性能.

\section{1 模式和资料简介}

SAMIL 是采用菱形截断的大气环流谱模式, 最 早版本系由林元弶从澳大利亚引进. 此后, 通过引入
参考大气、耦合简化的简单生物圈陆面模式 ${ }^{[15]}$ 等, 模 式在动力框架和物理过程方面都做了许多改进 ${ }^{[16 ~ 19]}$, 其中湿物理过程增加了 Tiedtke 方案, 替换了旧版本 的 Manabe 方案 ${ }^{[20]}$. 改进后 SAMIL 的基本模拟特征 表明, 模式可以模拟出孟加拉湾、印度半岛以及南海 主要的降水中心, 对东亚季风也有较好的模拟能力, 但是仍然存在一些问题, 主要表现为中低纬对流层 低层偏干，热带地区整层偏干偏冷，而降水明显偏多， 且存在“双赤道辐合带”(Double ITCZ)现象. 刘屹岷 等 ${ }^{[21]}$ 通过增加浅对流侧向混合的卷入卷出率, 同时 减小云水-雨水转换率，基本消除了 “双赤道辐合带” 现象, 同时改进了湿度和温度场的模拟. 尽管如此, 热带地区降水依然偏强. 上述现象表明引入 Tiedtke 方案后, 模式的湿物理过程还存在一些问题, 有必要 对改进前(Manabe 方案)与改进后(Tiedtke 方案)模式 的模拟性能作系统的评估.

本文采用 SAMIL 模式目前最高分辨率版本 R42L26 开展数值试验, 水平分辨率为 42 波截断, 相 当于 $2.8125^{\circ} \times 1.66^{\circ}$, 垂直方向分为 26 层, 采用 $\sigma-p$ 混 合坐标系. 辐射过程为 Edwards 和 Slingo ${ }^{[22]}$ 提出的辐 射参数化方案. 采用 Slingo $^{[23,24]}$ 提出的诊断云方案, 同时引入基于统计方法的层积云方案 ${ }^{[25]}$, 陆面过程 通过耦合 NCAR-CLM2.0 实现陆气通量交换 ${ }^{[26]}$. 此外, 模式还充分考虑了其他重要的次网格物理过程, 如 边界层参数化方案、重力波拖史等等. 关于 SAMIL 的详细介绍可参阅文献[27].

Manabe 对流调整方案假定当不稳定层结产生时, 对流使层结变为中性(式(1)), 且调整前后能量保持不 变(式(2)). Manabe 方案的缺点是忽略了环境空气的 卷入、云内空气的卷出等重要物理过程, 往往在对流 层低层产生过度降水.

$$
\begin{gathered}
\frac{\partial}{\partial p} \theta_{e}(\hat{T}, \hat{q}, p)=0, \\
\int_{p_{B}}^{p_{T}}\left[c_{p}(\hat{T}-T)+L(\hat{q}-q)\right] \mathrm{d} p=0,
\end{gathered}
$$

式中 $\theta_{e}, T$ 和 $q$ 分别表示湿位温、调整前的温度、比湿; $\hat{T}, \hat{q}\left(\hat{q}=p_{S}(\hat{T}, p)\right)$ 分别表示调整后的温度、比湿.

Tiedtke 方案是一种总体型质量通量积云参数化 方案 ${ }^{[5]}$, 该方案不考虑网格内的每一积云单体, 而仅 考虑与网格内所有积云单体上升、下沉气流等效的一 支总体上升、下沉气流的作用. 积云对流对大尺度收 
支方程的贡献可表示为

$$
\begin{aligned}
\frac{\partial \bar{s}}{\partial t}= & -\frac{1}{\bar{\rho}} \frac{\partial}{\partial z}\left[M_{u} s_{u}+M_{d} s_{d}-\left(M_{u}+M_{d}\right) \bar{s}\right] \\
& +L\left(c_{u}-e_{d}-\tilde{e}_{l}-\tilde{e}_{p}\right), \\
\frac{\partial \bar{q}}{\partial t}= & -\frac{1}{\bar{\rho}} \frac{\partial}{\partial z}\left[M_{u} q_{u}+M_{d} q_{d}-\left(M_{u}+M_{d}\right) \bar{q}\right] \\
& -\left(c_{u}-e_{d}-\tilde{e}_{l}-\tilde{e}_{p}\right),
\end{aligned}
$$

式中 $\bar{s}, \bar{q}$ 分别表示网格平均的干净力能、比湿; $M, s$, $q$ 分别表示积云的质量通量、干净力能及比湿, 下标 $u$ 对应云内上升气流, $d$ 对应云内下沉气流. $c_{u}, e_{d}, \tilde{e}_{l}$, $\tilde{e}_{p}$ 分别表示上升气流的凝结、下沉气流的蒸发、卷 出云水的蒸发、降水在未饱和次云层的蒸发. $L$ 为冰 水混合相的潜热系数.

为求解 $M_{u}$ 和 $M_{d}$, 需要得到云底质量通量 $M_{B}$, 一 般通过闭合假设得到. 对于深对流, 采用 Nordeng ${ }^{[28]}$ 于 1994 年提出的基于 CAPE 的调整型闭合方法:

$$
\begin{gathered}
\text { CAPE }=\int\left(\frac{g}{\bar{T}_{v}}\left(T_{v}-\bar{T}_{v}\right)-g l\right) \mathrm{d} z, \\
\frac{\partial \mathrm{CAPE}}{\partial t} \approx-\int \frac{g}{\bar{T}_{v}} \frac{\partial \bar{T}_{v}}{\partial t} \mathrm{~d} z \\
\approx-M_{B} \int\left(\frac{(1+\delta \bar{q})}{c_{p} \bar{T}_{v}} \frac{\partial \bar{s}}{\partial z}+\delta \frac{\partial \bar{q}}{\partial z}\right) \eta \frac{g \mathrm{~d} z}{\bar{\rho}},
\end{gathered}
$$

其中, $T_{v}$ 和 $\bar{T}_{v}$ 分别表示积云和环境的虚温, $\eta$ 为归一化 的质量通量. 实际计算时, 首先通过初估的云底质量 通量 $M_{B}^{*}$ 得到 $\eta$, 然后按式(6)重新计算出 $M_{B}$.

对于浅对流，闭合假设基于次云层水汽收支平 衡得到云底质量通量 $M_{B}$; 中层对流的云底质量通量 取决于大尺度上升气流产生的垂直质量输送.

Tiedtke 方案的触发条件为: 如果从地面沿干绝 热线抬升的未稀释气块可以逐层保持正浮力并上升 至抬升凝结高度层, 则可以发生深对流或浅对流. 如 果不能发生深对流或浅对流, 则考虑中层对流, 即从 模式底部 3 10 层中寻找相对湿度大于 90\%的层次作 为中层对流云底层, 若云底之上存在对流不稳定层 且有大尺度上升运动，则触发中层对流.

由上可见, Tiedtke 方案对大尺度强迫作用和调 整过程都作了细致处理, 而 Manabe 方案中的对流调 整实际上是瞬时调整, 忽略了调整过程的影响.

模式采用的层状降水方案是由 Manabe 等 $^{[2]}$ 于 1965 年提出的大尺度调整方案, 对湿度达到饱和且
湿对流稳定的点, 进行大尺度调整.

调整后的比湿为

$$
\hat{q}_{k}=q_{k}^{*}+\frac{\mathrm{d} q_{k}^{*}}{\mathrm{~d} T}\left(\hat{T}_{k}-T_{k}\right) ;
$$

调整后的温度为

$$
\hat{T}_{k}=T_{k}+\frac{L}{c_{p}}\left(q_{k}-\hat{q}_{k}\right) .
$$

由式(7)和式(8)联立得到

$$
\hat{q}_{k}=\left(q_{k}^{*}+q_{k} \frac{L}{c_{p}} \frac{\mathrm{d} q_{k}^{*}}{\mathrm{~d} T}\right) /\left(1+\frac{L}{c_{p}} \frac{\mathrm{d} q_{k}^{*}}{\mathrm{~d} T}\right),
$$

式中, $T_{k}, q_{k}, q_{k}^{*}$ 为调整前的温度、比湿、饱和比湿; $\hat{T}_{k}$ 和 $\hat{q}_{k}$ 分别为调整后的温度和比湿, $l$ 为水的相变潜 热, $c_{p}$ 为干空气比定压热容, 下标 $k$ 表示模式的第 $k$ 层.

模式在逐年月平均海温强迫下，分别采用 Manabe 和 Tiedtke 两种对流方案各积分 $25 \mathrm{a}$, 取后 10 $\mathrm{a}$ 平均作为模拟结果. 用于检验模式结果的资料有: (1) GPCP(Global Precipitation Climatology Project)降 水资料 ${ }^{[29]}$; (2) CMAP 降水资料 ${ }^{[30] ;}$; (3) TRMM-3A12 (Tropical Rainfall Measuring Mission); (4) TRMM3A25; (5) 欧洲中期天气预报中心的 ERA40 再分析 资料 ${ }^{[31]}$; (6) NCEP/NCAR 再分析资料 ${ }^{[32]}$.

\section{2 降水场}

降水场的模拟是检验对流参数化方案优劣的主 要指标, 同时也是数值模式动力框架和物理过程综 合作用的结果. 为客观评估 Manabe 方案和 Tiedtke 方案对降水的模拟能力, 利用 TRMM 的两类产品 TRMM-3A12, TRMM-3A25 作为检验标准, 其中 $3 \mathrm{~A} 12$ 是 TRMM 卫星微波成像仪(TMI)的观测结果, $3 \mathrm{~A} 25$ 是星载降水雷达 $(\mathrm{PR})$ 的观测结果. 图 1 给出了 观测和模拟的热带地区夏季对流降水分布. 由图 1(a) 和(b)可见, TRMM观测主要表现为位于北赤道辐合 带(ITCZ)的强降水, 最大降水中心位于孟加拉湾, 其 次为赤道东太平洋、南海、印度半岛以及西太平洋暖 池，大部分都是对流云团盛行的季风区，赤道以南的 降水则较弱. 另外, 对比图 1(a)和(b), 发现 3A25 揭 示的对流降水强度小于 $3 \mathrm{~A} 12$, 大约为 $3 \mathrm{~mm} / \mathrm{d}$. 由图 1(c)和(d)可知, Tiedtke 方案能很好地模拟出季风区的 降水中心, 如孟加拉湾、印度半岛等等, 但模拟的菲 

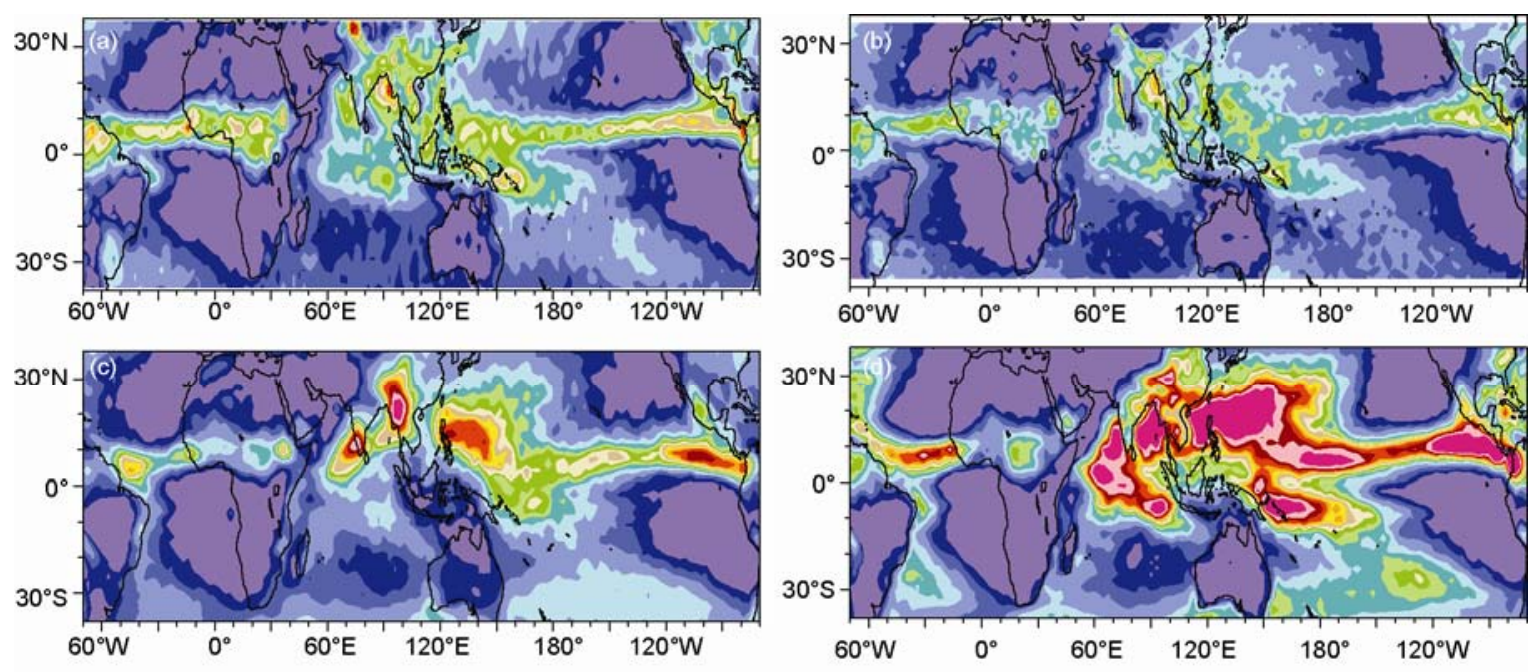

$\begin{array}{lllllllllllllll}0.2 & 0.5 & 1 & 2 & 3 & 4 & 5 & 6 & 7 & 8 & 9 & 10 & 12 & 14 & 17(\mathrm{~mm} / \mathrm{d})\end{array}$

图 1 观测和模拟的热带地区夏季(JJA)对流降水分布

(a) TRMM-3A12; (b) TRMM-3A25; (c) Manabe 方案模拟; (d) Tiedtke 方案模拟

律宾降水中心位置偏北, 且范围过大. 与此相比, Manabe 方案模拟的菲律宾降水中心同观测基本一致, 但模拟的孟加拉湾降水中心较观测明显向东向北伸 展, 在高原下游出现虚假的强降水. 就降水强度而言, 两种方案的模拟结果均较观测偏强, 相比之下, Manabe 方案的模拟结果与观测更为接近. 导致上述 结果的原因可能有三个: (1) Tiedtke 方案的对流触发 条件比较严格, 不利于弱的对流频繁发生, 而利于对 流不稳定能量的积累, 强不稳定能量释放对应着强 的对流降水; (2) 模式中层状降水过程通过改变温 度、湿度的空间分布, 从而对下一时步的对流过程 产生间接影响; (3) 缺乏海气耦合过程使洋面降水偏 强 ${ }^{[33]}$.

图 2 给出的是观测和模拟的热带地区夏季层状 降水分布，观测表明雨带分布的地理位置同对流降 水极为相似, 主要位于赤道辐合带(ITCZ)、季风区以 及西太平洋暖池. 对于热带地区出现的层状降水, Houz $\mathrm{e}^{[34,35]}$ 认为此类层状降水与对流降水是同一块积 雨云在生命期的不同阶段形成的，层状降水通常出 现在积雨云的衰弱期, 对流层低层为下沉运动控制, 而上层为弱上升运动, 降水粒子的增长主要通过水 汽凝华, 在雷达拼图上呈现出层状结构的降水回波. 由图 2(c) 可见, Manabe 方案较好地模拟出主要的层状 降水中心, 且地理分布特征同对流降水类似, 表明层
状降水主要出现在热带对流区, 这与 TRMM 的观测 结果一致. 图 2(d) 是 Tiedtke 方案的模拟结果, 与 TRMM 相比, 其模拟的层状降水明显失真, 大于 3 $\mathrm{mm} / \mathrm{d}$ 的雨区基本没有, 另外也没有模拟出沿赤道的 降水中心, 微量降水主要分布在赤道以外太平洋、大 西洋的东海岸.

两种方案下大洋东岸层状降水模拟的误差可能 与模式的低云方案有关, 夏季大洋东海岸受副热带 高压控制, 盛行下沉气流, 大气稳定度高, 在对流层 低层形成明显的逆温层. 又由于海水的蒸发不断向 上层输送水汽, 因此卫星云图上常出现低云. 由于模 式目前采用的仍是诊断云方案，云没有通过显示方 式纳入水循环的体系，因此在大气既稳定又饱和的 情况下触发了层状降水的启动机制.

对比图 1(d)和 2(d), 发现 Tiedtke 方案模拟的对 流降水偏强, 层状降水偏弱, Manabe 方案模拟的两类 降水强度基本一致, 揭示层状降水与对流降水的比 例可以进一步反映两种对流方案在降水模拟上的差 异. 图 3 给出的是观测和模拟的夏季 $(J J A)$ 、冬季 (DJF)、全年(ANN)层状降水占总降水比例的空间分 布. 由图 3(a) (f) 可知, 在副热带地区, TRMM 揭示的 层状降水比例达到 $50 \%$ 以上, 这是由于赤道以外地 区冷暖空气交绥频繁, 锋面降水占了很大比重. 在赤 道地区，无论冬季还是夏季，观测的层状降水比例达 

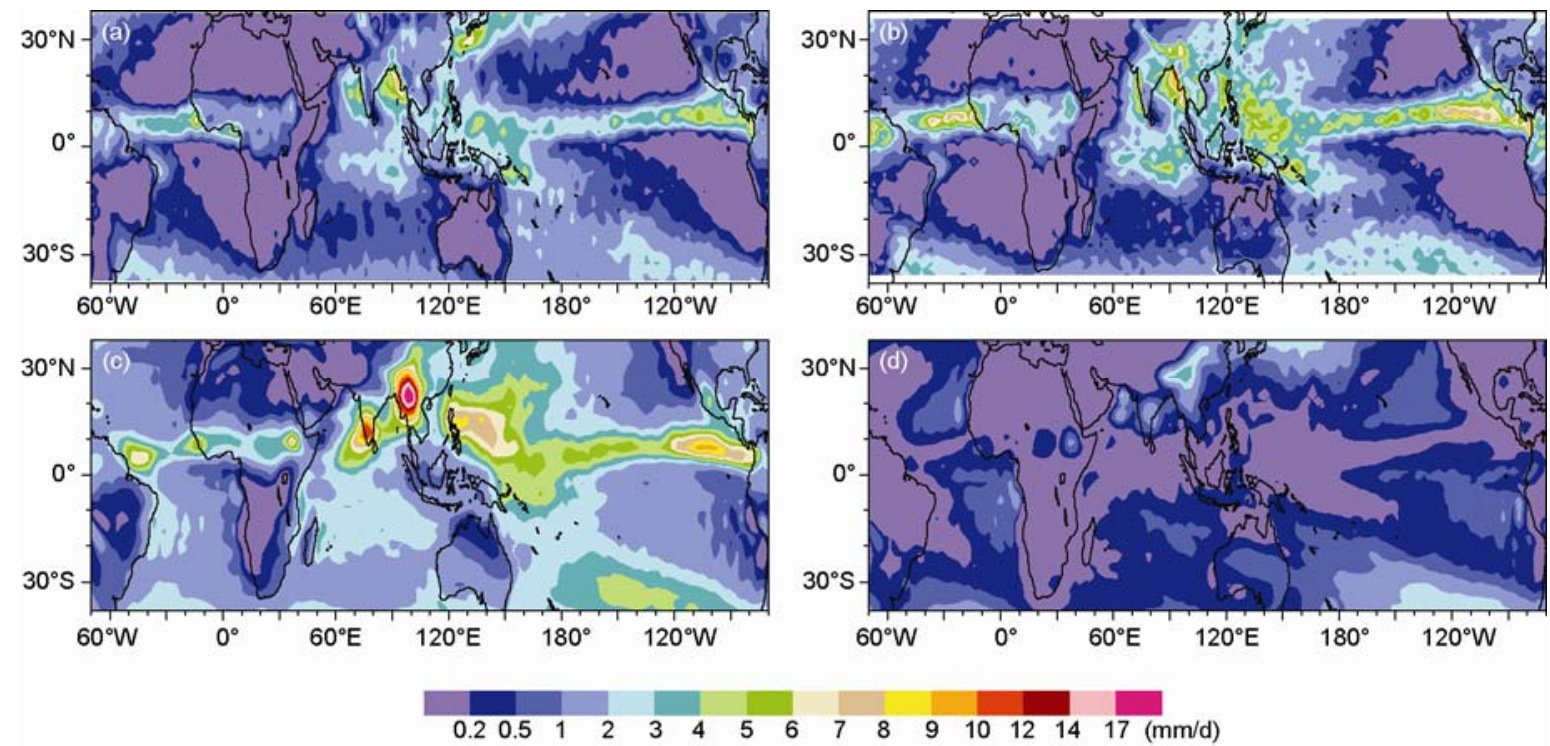

图 2 观测和模拟的热带地区夏季 $(\mathbf{J J A})$ 层状降水分布

(a) TRMM-3A12; (b) TRMM-3A25; (c) Manabe 方案模拟; (d) Tiedtke 方案模拟
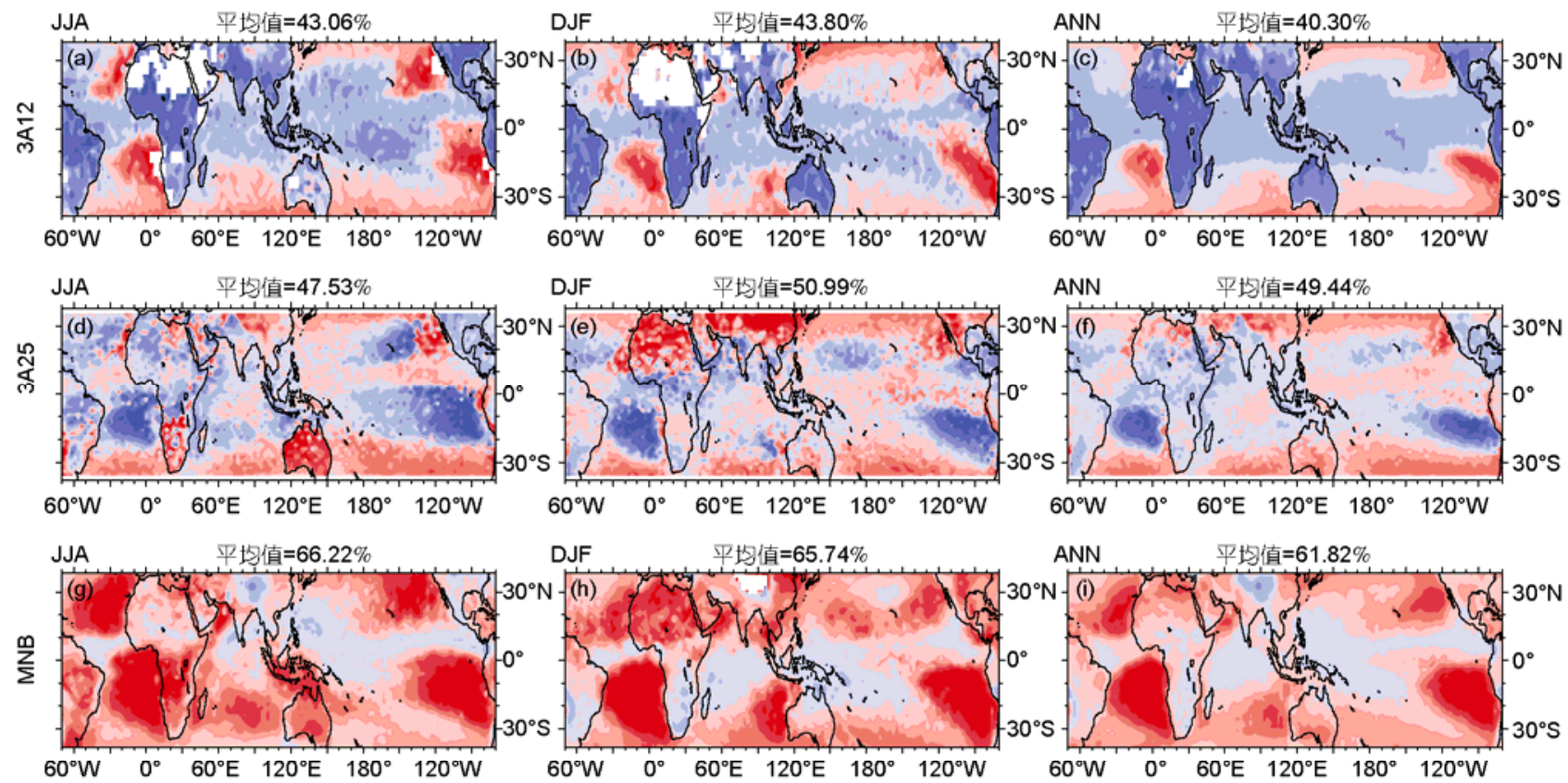

DJF 平均值 $=65.74 \%$
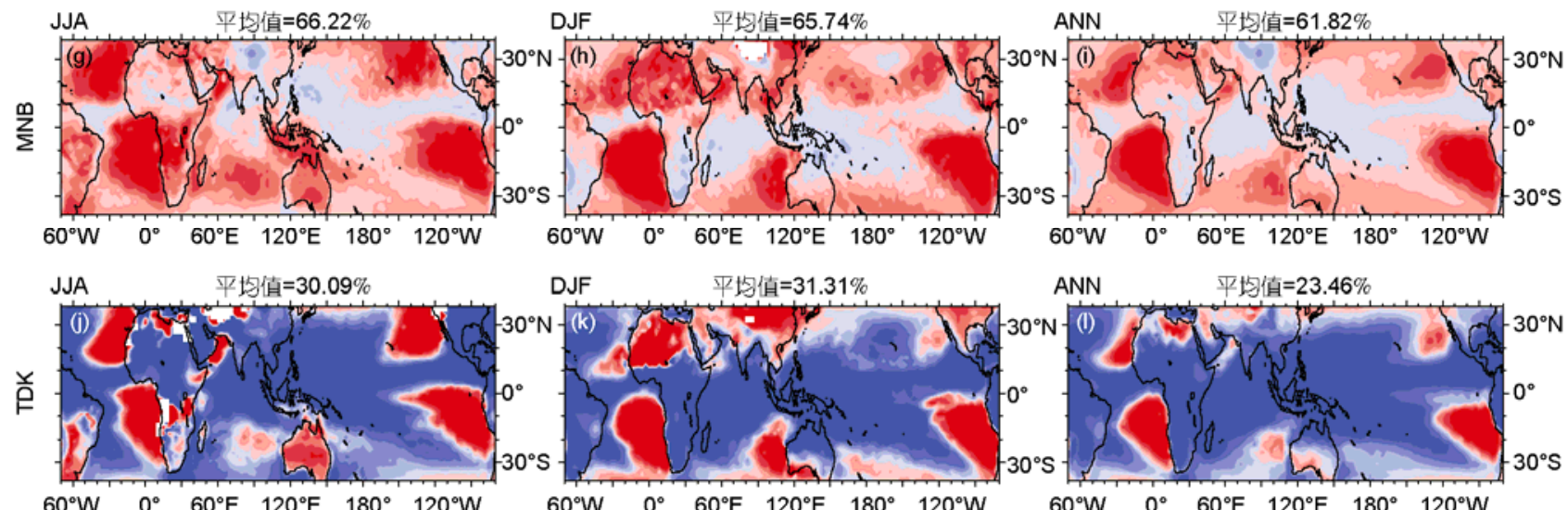

ANN 平均值 $=23.46 \%$

$60^{\circ} \mathrm{W} \quad 0^{\circ} \quad 60^{\circ} \mathrm{E} \quad 120^{\circ} \mathrm{E} \quad 180^{\circ} \quad 120^{\circ} \mathrm{W}$

$60^{\circ} \mathrm{W} \quad 0^{\circ} \quad 60^{\circ} \mathrm{E} \quad 120^{\circ} \mathrm{E} \quad 180^{\circ} \quad 120^{\circ} \mathrm{W}$

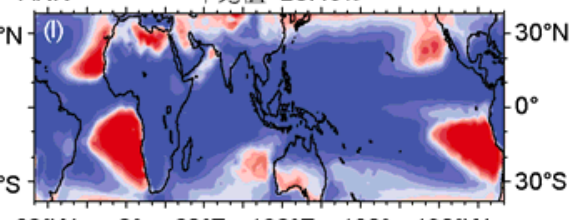

10

20

$30 \quad 40 \quad 50 \quad 60$

80

$90(\%)$

图 3 观测和模拟的层状降水比例空间分布

(a) (c) TRMM-3A12; (d) (f) TRMM-3A25; (g) (i) Manabe 方案模拟; (j) (l) Tiedtke 方案模拟 
到了 $40 \%, 3 \mathrm{~A} 25$ 揭示的比例甚至更高, 接近 50\%.

最初人们认为层状降水仅出现在中纬度气旋与 锋面系统中, 因为那里大气以弱上升运动为主, 降水 雷达回波表现为层状结构. 而热带是对流活动区, 因 而认为热带地区的降水为对流性降水. 1974年开展的 GATE 试验发现：热带云系中同样有层状降水回波存

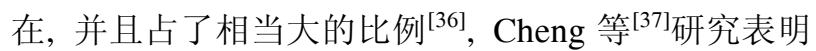
热带洋面上的层状降水占总降水的 $40 \%$ 以上. 图 3(g) (1)是 Manabe 方案和 Tiedtke 方案模拟的结果. 由图 3 可知, Manabe 方案模拟的层状降水比例在热带 洋面上接近观测, 大约为 $40 \%$ 50\%, 介于 $3 \mathrm{~A} 12$ 与 $3 \mathrm{~A} 25$ 之间, 而在副热带地区明显高于观测, 达到 $90 \%$ 以上. 与此相比, Tiedtke 方案由于模拟的层状降 水偏弱、对流降水偏强, 致使大部分地区层状降水比 例都低于 $10 \%$.
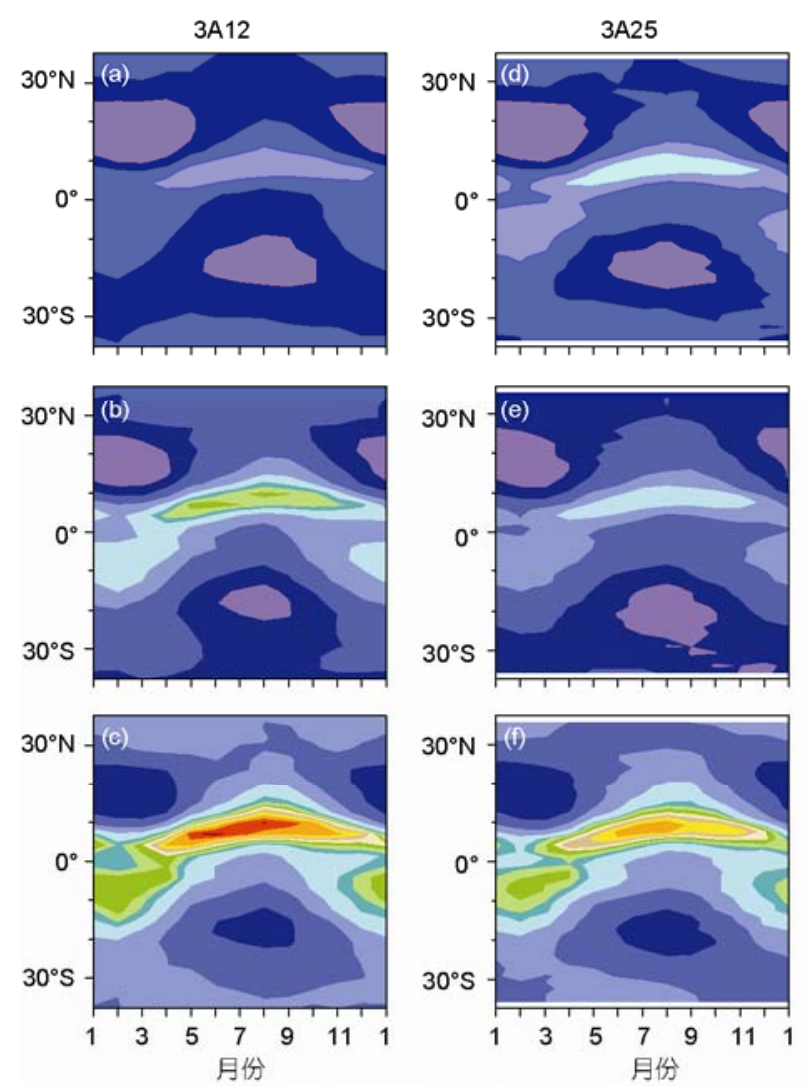

对于 Tiedtke 方案下层状降水与对流降水比例失 调的问题, 起初以为积云对流过程中不稳定能量的 释放消耗了过多的水汽, 使环境大气偏干, 从而导致 层状降水偏少. 然而, 即使调换模式中降水模块的先 后顺序，先计算层状降水后计算对流降水，结果并无 改善. 最终, 经过研究比较 ECHAM 模式 ${ }^{[38]}$ 的湿物理 过程，发现问题的根源出在层状降水方案上. 稳定凝 结方案本质上是一种调整过程, 没有考虑 Tiedtke 对 流过程中卷出的云水、云冰等水物质，而卷出的这部 分水物质能够用来增湿环境大气或直接形成降水. 换句话说，模式采用的层状降水方案同 Tiedtke 方案 不匹配, 与 Manabe 方案则是匹配的, 因为两者都是 基于湿静力能守恒的调整方案, Manabe 方案对层状 降水的模拟之所以有更合理的结果也源于此.

图 4 给出的是纬向平均后观测和模拟的层状降
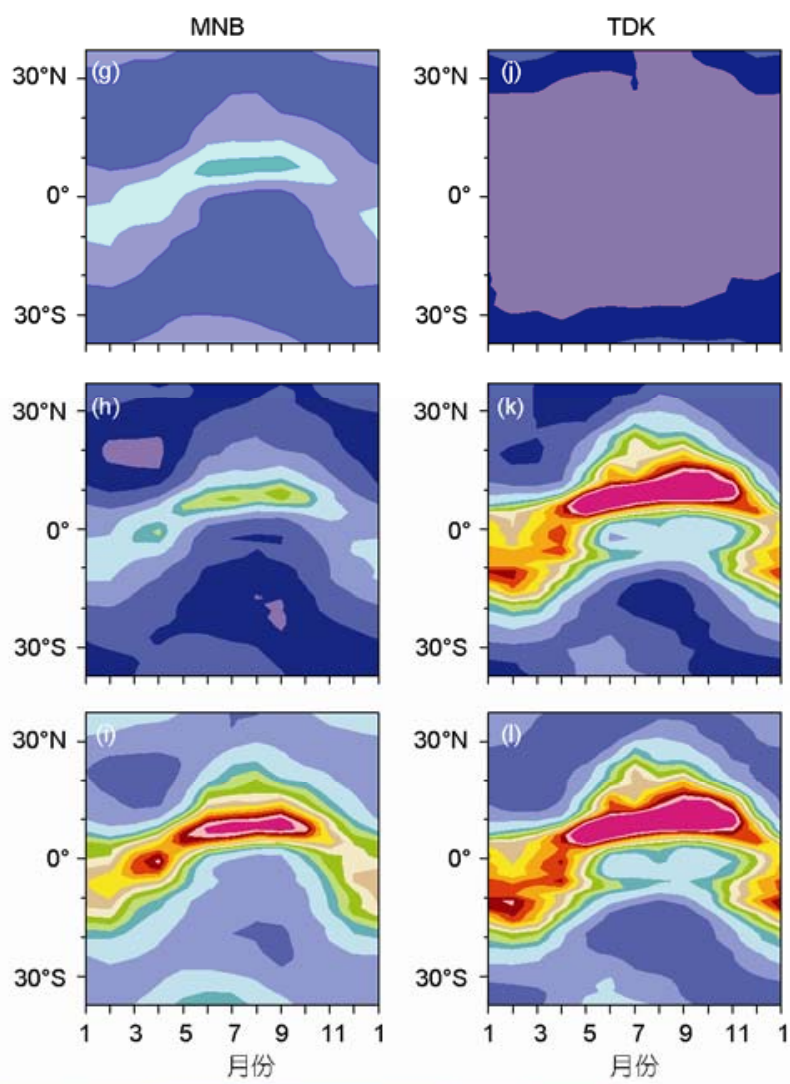

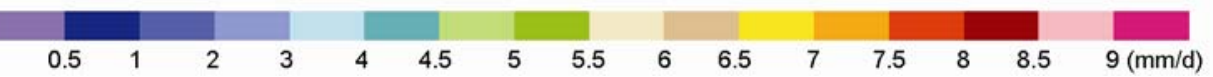

图 4 纬向平均的层状降水、对流降水、总降水的时空分布

(a) (c) TRMM-3A12; (d) (f) TRMM-3A25; (g) (i) Manabe 方案模拟; (j) (l) Tiedtke 方案模拟. (a), (d), (g), (j)为层状降水; (b), (e), (h), (k)为对流 降水; (c), (f), (i), (1)为总降水 
水、对流降水以及总降水的时间-纬度图. 由图 4(a) (f) 可知，TRMM 资料揭示的层状降水与对流降水有一 致的时空分布, 表现为春季雨带随季节北移, 在夏季 达到最北, 同时强度达到最大, 此后雨带随季节南撤, 在冬季回到赤道以南, 至此完成降水的年循环. 经对 比发现, TRMM 两种资料揭示的降水强度有一定差 异, 3A12 揭示的总降水、对流降水强于 $3 \mathrm{~A} 25$, 而层 状降水则弱于 $3 \mathrm{~A} 25$. 就 TRMM 同一产品内的两类降 水而言, $3 \mathrm{~A} 12$ 的对流降水明显强于层状降水, 而 $3 \mathrm{~A} 25$ 中两类降水强度基本相同, 这与图 3(a) (f)所示 情况一致. 由图 4(g) (l)可知, 对于对流降水和总降 水, Manabe 方案和 Tiedtke 方案都较好地再现了雨带 随季节的南北移动, 但降水强度均偏强, 尤其是 Tiedtke 方案. 而对于层状降水, Tiedtke 方案由于模 拟的层状降水失真, 模拟不出合理的年循环, 与之相 比, Manabe 方案的模拟结果与观测较为接近.

\section{3 凝结潜热加热}

非绝热加热是大气运动及变化的主要能源. 在 热带地区, 由降水引起的凝结潜热释放是其主要部
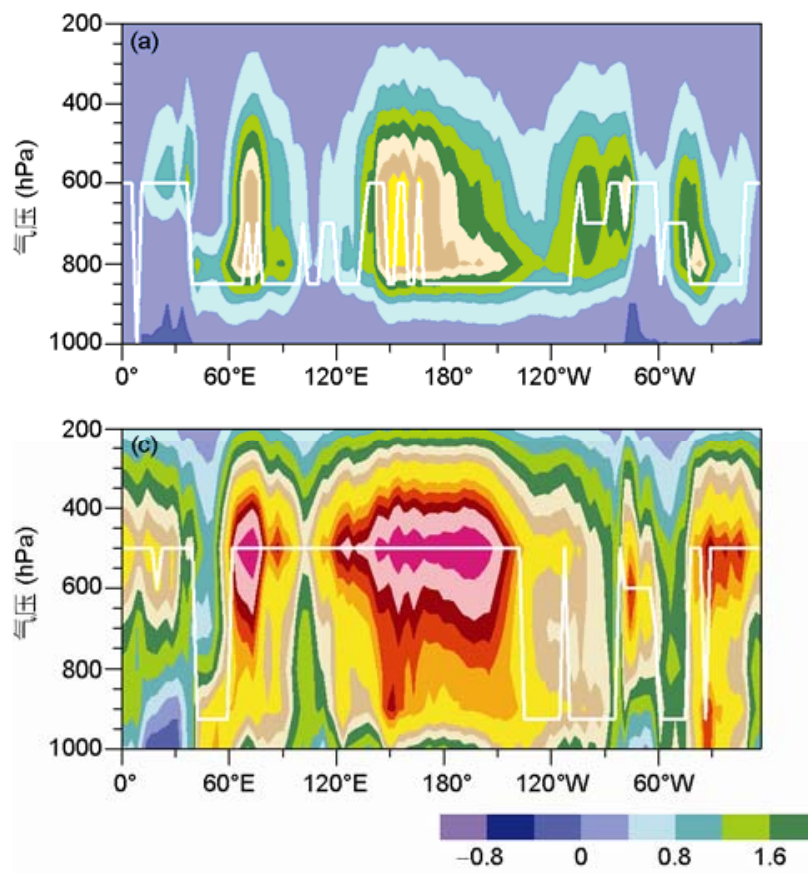

分. 凝结潜热对副热带高压的形成和变异 ${ }^{[39]}$ 、热带地 区大气低频振荡有重要影响. 图 5 给出了两种对流方 案模拟的赤道地区 (取 $5^{\circ} \mathrm{S} 5^{\circ} \mathrm{N}$ 经向平均)对流加热及 层状加热垂直剖面图. 由图 5(a)和(c)可知, 两种方案 模拟的对流凝结潜热释放中心大致位于印度季风区 以及赤道辐合带, 这与此两个地区盛行对流降水是 一致的. 注意, 这里的对流凝结潜热指的是整个对流 过程的加热率, 因此, 对于 Tiedtke 方案, 除相变引起 的加热外, 还包括了温度扰动的垂直输送. 由于 Tiedtke 方案模拟的对流降水较 Manabe 方案偏多, 因 而对应的凝结潜热释放也偏强. 另外 Tiedtke 方案中 最大凝结加热所在高度位于 $500 \mathrm{hPa}$, 而 Manabe 方案 中最大凝结加热所在高度位于 $600 \mathrm{hPa}$ 以下. 图 5(b) 和(d)分别是 Manabe 方案、Tiedtke 方案模拟的层状 凝结加热, 由图可知, Manabe 方案模拟的层状加热强 度与对流加热强度相似, 这同层状降水与对流降水 间的比例是一致的，最大凝结加热所在高度位于 400 $\mathrm{hPa}$. 值得注意的是, 在洋面上低层 $900 \mathrm{hPa}$ 处出现了 强的加热中心，这与实际观测是不相符的，卫星观测 表明与层状降水相伴的加热剖面表现为冻结层 $\left(0^{\circ} \mathrm{C}\right.$ 层)之上加热，其下冷却. Tiedtke 方案模拟的层状加
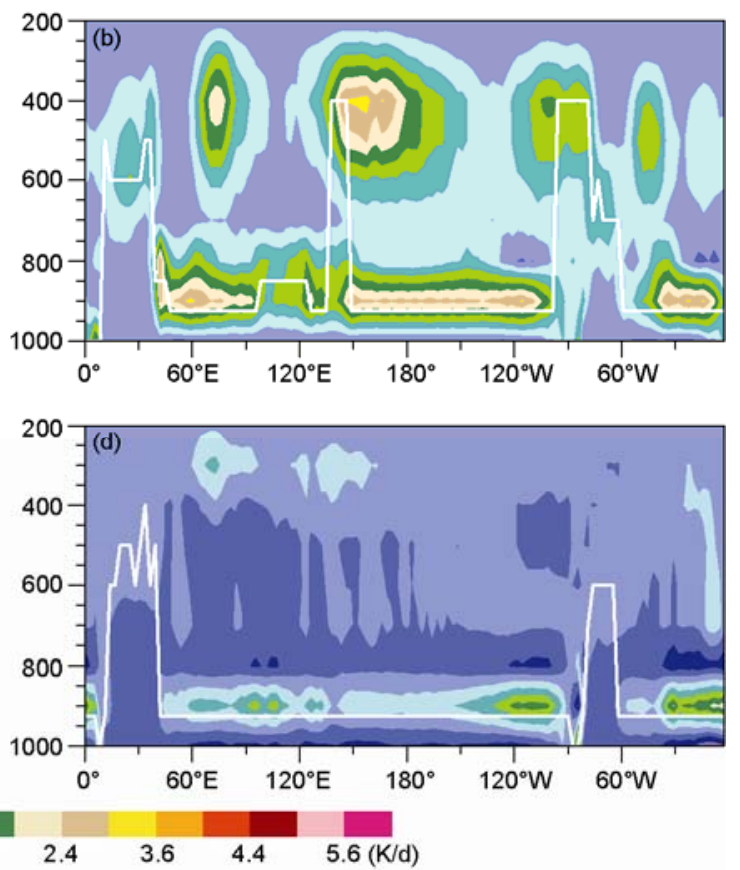

图 5 夏季 $(\mathrm{JJA})$ 凝结潜热加热经向平均 $\left(5^{\circ} \mathrm{S} 5^{\circ} \mathrm{N}\right)$ 垂直剖面图

(a) Manabe 方案模拟的对流凝结加热; (b) Manabe 方案模拟的层状凝结加热; (c) Tiedtke 方案模拟的对流凝结加热; (d) Tiedtke 方案模拟的层状 凝结加热. 图中白色折线表示最大凝结加热所在层次 
热偏弱, 高层加热中心不明显, 在低层 600 800 hPa 处为冷却, $800 \mathrm{hPa}$ 以下同样有加热中心出现. 对流 层低层出现的虚假加热一方面与模式的湿物理过程 有关, 另一方面可能由于模式未充分考虑降水在洋 面上的蒸发.

积云对流凝结加热反馈理论是 MJO 形成及维持 的主要动力学机制 ${ }^{[40]}$, 凝结加热垂直廓线对 MJO 信 号的强度与传播有重要影响. Lin 等 ${ }^{[14]}$ 通过 IFA 增强 观测场表明, MJO 湿位相期, 对流凝结加热表现为整 层加热, 最大加热高度位于 $500 \mathrm{hPa}$ 附近; 层状凝结 加热在 $0^{\circ} \mathrm{C}$ 之上表现为加热, 其下表现为冷却. 图 6(a) 和(b) 是 Manabe 方案模拟的 MJO 湿位相期(日降水大 于 $4 \mathrm{~mm})$ 西太平洋 $\left(5^{\circ} \mathrm{N} 5^{\circ} \mathrm{S}, 145^{\circ} \mathrm{E} 155^{\circ} \mathrm{E}\right)$ 对流加热 与层状加热的垂直廓线. 对流加热在 $900 \mathrm{hPa}$ 以上表 现为整层加热, 最大加热所在高度为 $700,900 \mathrm{hPa}$ 以 下表现为弱冷却; 而对于层状凝结加热, Manabe 方 案没有模拟出 $0^{\circ} \mathrm{C}$ 层之下的冷却, 而表现为整层加热,
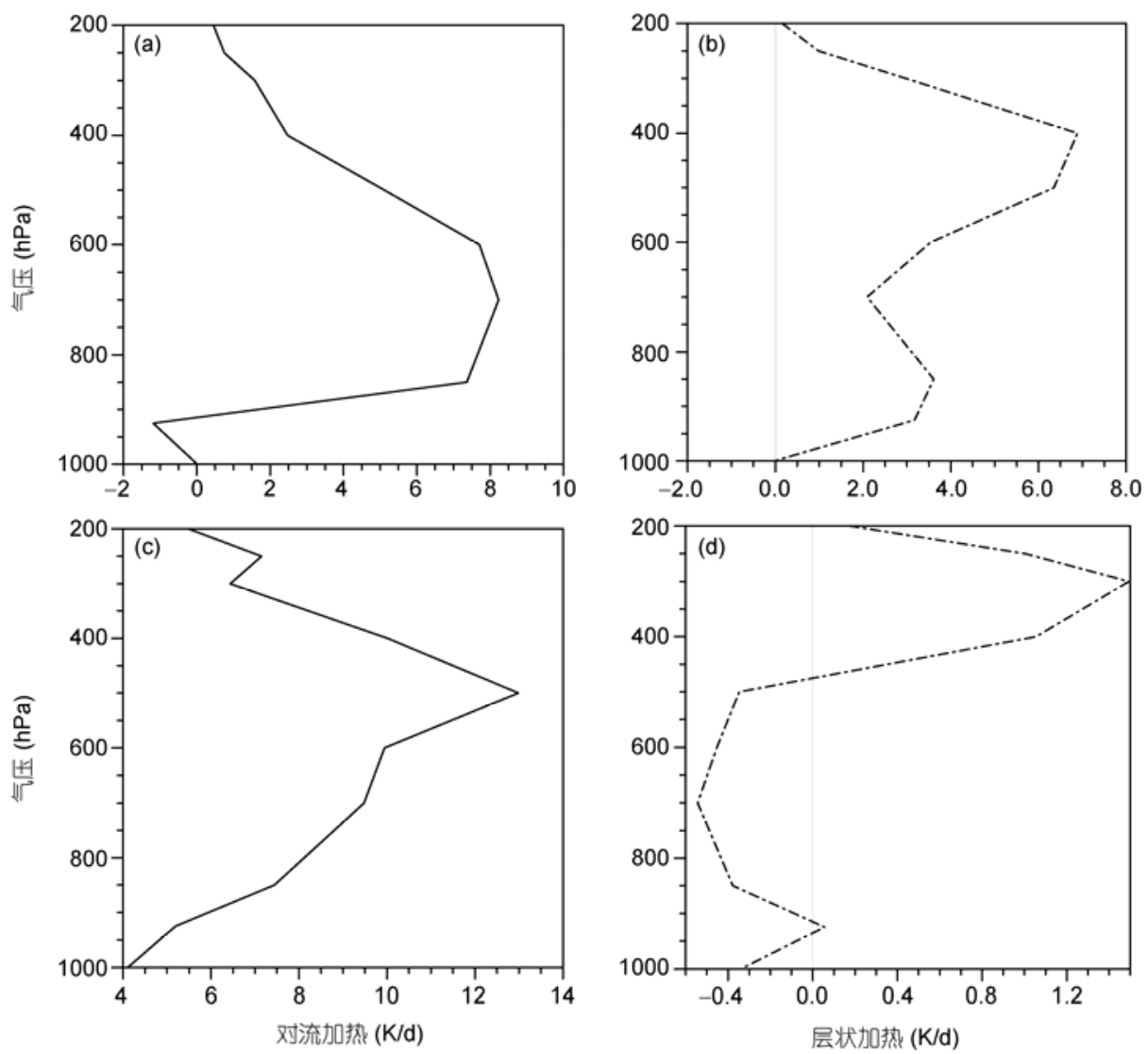

图 6 MJO 湿位相期凝结加热垂直廓线

(a) MNB 模拟的对流加热; (b) MNB 模拟的层状加热; (c) TDK 模拟的对流加热; (d) TDK 模拟的层状加热 
又同水汽、温度等气象要素场紧密联系, 因此有必要 通过比较模拟的环流场, 以便更全面地评估两种对 流方案.

Taylor 图 ${ }^{[41]}$ 可以定量地说明模式模拟的要素场 与观测场的空间相似度, 图 7 给出了两种对流方案模 拟的气象要素 Taylor 图. 图 7(a)和(b)是冬、夏季节 Manabe 方案与 Tiedtke 方案的比较结果, 选用的基准 资料为 ERA40. 图中点距原点的距离 (半径)表示标准 化后模拟和观测的比率, 与纵坐标的角度表示模式 和观测之间空间相关系数的反余弦. 离图中 REF 所 示位置越近, 说明模拟越接近观测.

由图 7 可知, 冬季, 无论标准差还是相关系数, Tiedtke 方案的模拟结果与 ERA40 的相似度更好, 尤 其对于对流层高层 $200 \mathrm{hPa}$ 温度, Tiedtke 方案模拟的

(a)

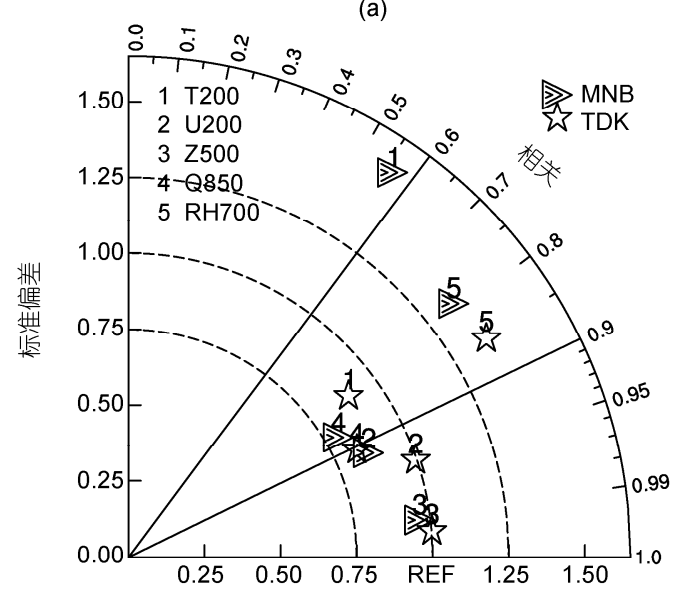

(c)

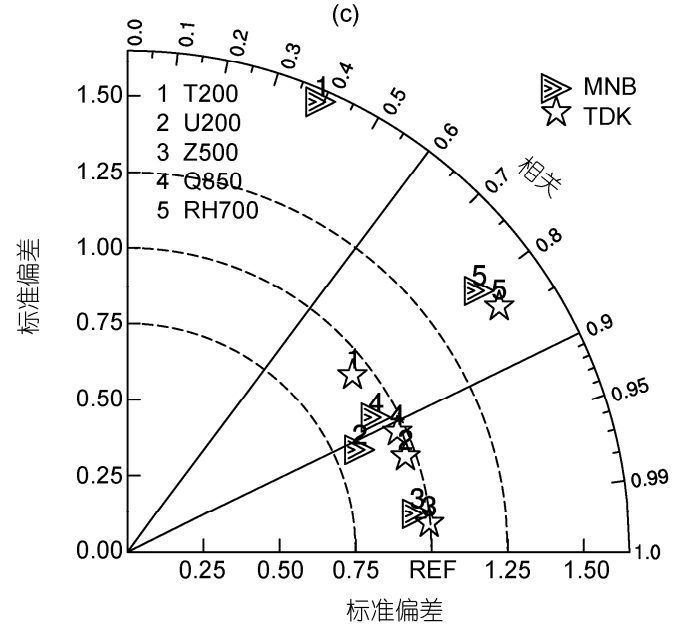

相关系数接近 $80 \%$, 与再分析资料的标准差比率在 1.0 附近, 而 Manabe 方案模拟的相关系数不足 $60 \%$, 且标准差比率达到 1.5. 对流层高层温度主要受云-辐 射过程影响, 云量由湿度场诊断得到, 而湿度场很大 程度上取决于对流过程, 即潜热加热空间结构的模 拟. 由第 3 节可知, Tiedtke 方案模拟的潜热加热空间 结构优于 Manabe 方案, 因而对流层高层温度也模拟 的更为合理. 夏季, 两种对流方案的模拟结果较为接 近, 对于 $200 \mathrm{hPa}$ 纬向风的模拟, Manabe 方案略好一 些, 但整体上仍逊于 Tiedtke 方案. 图 7(c)和(d)是基 准资料为 NCEP 的 Taylor 图, 所示情况与图 7(a)和(b) 类似，表明 ERA40, NCEP 再分析资料描述的气象要 素场基本一致. 对比图 7(b)和(d)发现, NCEP 揭示的 $850 \mathrm{hPa}$ 比湿标准差要小于 ERA40, 且二者与模式的

(b)

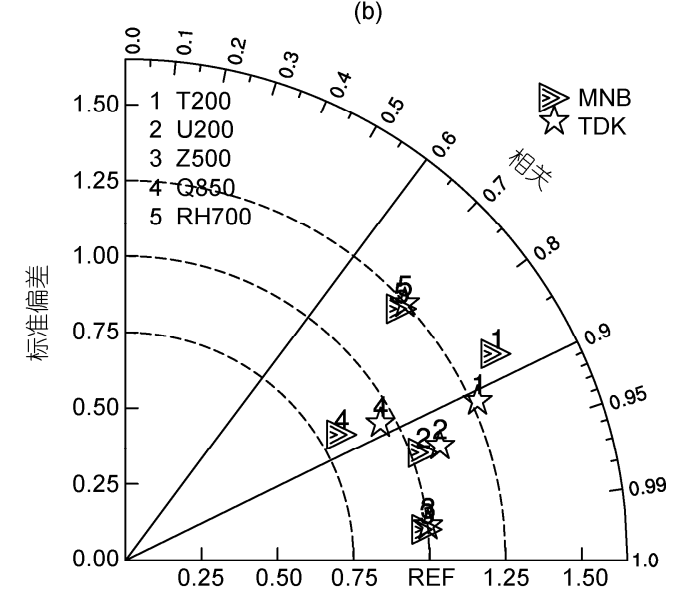

(d)

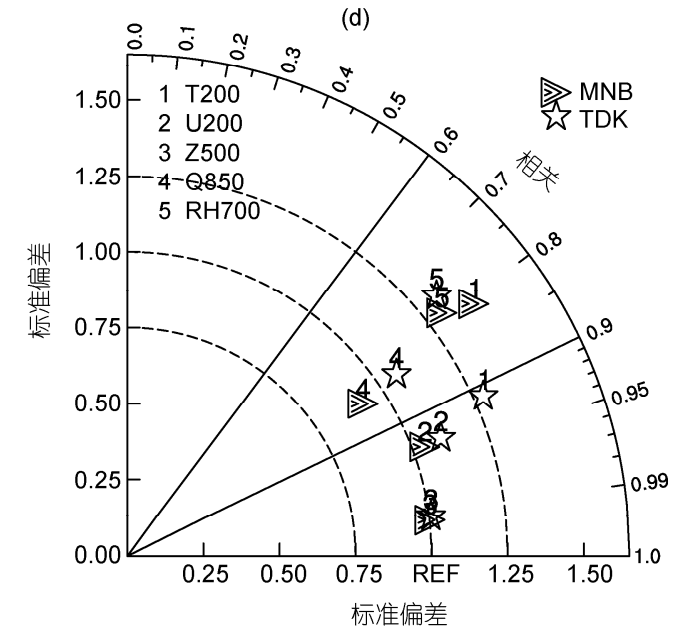

图 7 两种对流方案模拟的气象要素 Taylor 图

(a) 冬季模拟结果(REF: ERA40); (b) 夏季模拟结果(REF: ERA40); (c) 冬季模拟结果(REF: NCEP); (d) 夏季模拟结果(REF: NCEP) 
相关系数也略有差异. 这表明, 水汽作为湿物理过程 的关键要素, 即使在再分析资料中也有很大的不确 定性.

\section{5 敏感性试验}

Tiedtke 方案模拟的对流降水偏强, 层状降水偏 弱, 可能与不匹配的层状降水方案有关, 即在层状降 水方案中忽略了积云对流过程中卷出的云水、云冰, 而卷出的这部分水凝物对层状降水是有贡献的. 为 证实这一想法, 我们进行了敏感性试验, 即将卷出的 云水(云冰)全部蒸发(升华)用于增湿环境大气, 再调 用层状降水过程, 得到的模拟结果如图 8 所示, 试验 成功模拟出了沿赤道辐合带(ITCZ)的层状降水, 并 且层状降水比例得到大幅提高. 另外, 考虑卷出云水 (云冰)后, 同样能模拟出峰值位于 $500 \mathrm{hPa}$ 的对流加 热, 以及“top-heavy”的层状加热结构, 并且由于层 状降水比例增加, 对应的加热强度也得到一定改善 (图略).

\section{6 结论和讨论}

本文基于 TRMM 卫星资料和 ERA40, NCEP 再 分析资料, 对 LASG/IAP 新版大气环流模式 SAMIL 中 Manabe, Tiedtke 两种对流方案的模拟结果进行了 分析评估, 主要结论如下:
(1) Tiedtke 方案模拟的对流降水偏强, 尤其是位 于菲律宾以北的西太平洋地区, 层状降水显著偏弱, 没有能模拟出沿赤道的降水中心. 与之相比, Manabe 方案的模拟结果与观测较为接近, 但其模拟的孟加 拉湾降水中心向东向北伸展, 在高原下游有虚假强 降水出现. 从层状降水所占比例来看, 在赤道洋面上, Manabe 方案的模拟结果介于 TRMM-3A12 与 TRMM-3A25 之间, 大约占 40\% 50\%. 而在赤道以外 地区，则明显高于观测，达到 $90 \%$ 以. Tiedtke 方案 由于模拟的对流降水偏强、层状降水偏弱, 使得层状 降水比例低于观测. 降水的时空分布亦表明: Tiedtke 方案由于模拟的层状降水失真, 因而不能合理再现 雨带随季节的南北移动.

(2) Tiedtke 方案虽然对降水的模拟不如 Manabe 方案，但其模拟的凝结潜热的垂直结构则远较 Manabe 方案合理, 表现为与观测一致的位于对流层 中层 $500 \mathrm{hPa}$ 处的对流加热峰值, 以及 $0^{\circ} \mathrm{C}$ 层之上的 层状加热与其下的冷却. 与此相比, Manabe方案模拟 的最大对流加热所在高度较观测偏低, 大致位于 700 $\mathrm{hPa}$. 对于层状加热, Manabe 方案模拟不出 $0^{\circ} \mathrm{C}$ 层以 下的冷却, 而表现为整层加热.

(3) 温度、比湿等要素的 Taylor 图表明: Tiedtke 方案的模拟结果与 ERA40, NCEP 再分析资料的相关 系数更高, 尤其是对流层 $200 \mathrm{hPa}$ 温度的模拟.

(4) Manabe 方案能在较不合理的垂直加热结构
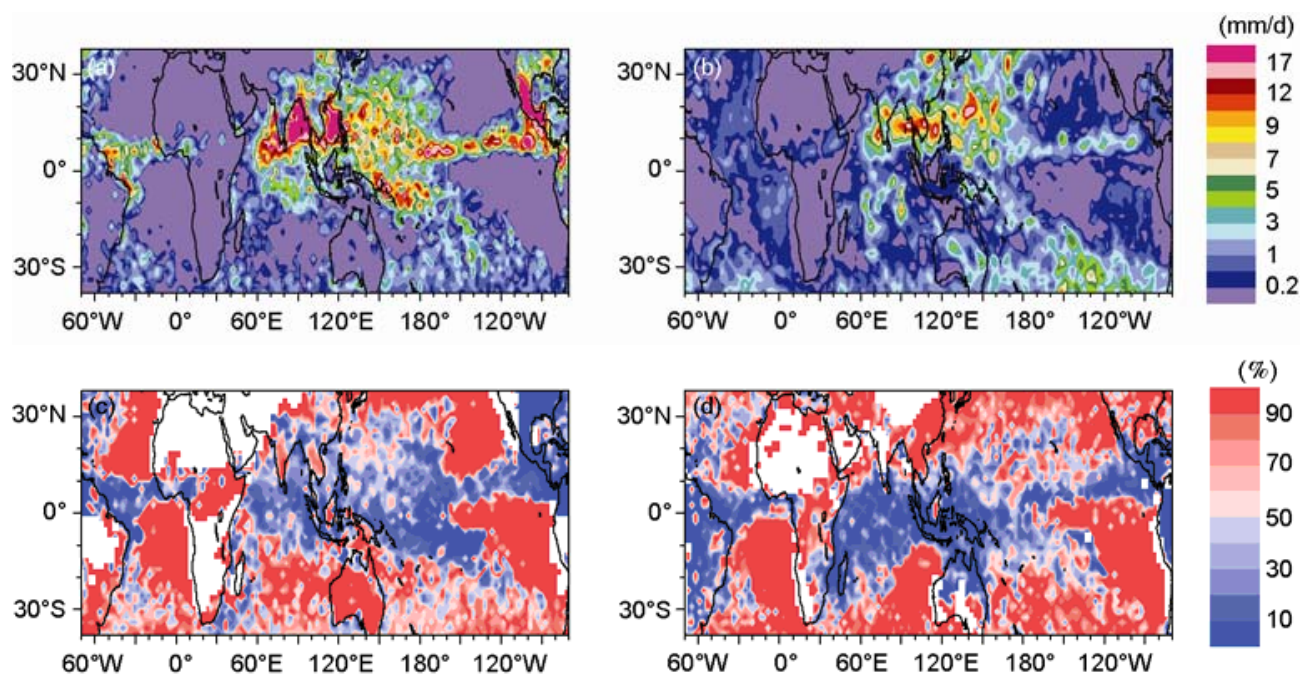

图 8 敏感性试验结果

(a) 对流降水分布 (JJA); (b) 层状降水分布(JJA); (c) 层状降水比重 $(\mathrm{JJA})$; (d) 层状降水比重(DJF) 
和环流场下模拟出相对合理的降水, 很大程度上与 Manabe 方案的相对简单有关, 其触发只取决于饱和 和湿对流不稳定两个条件, 而 Tiedtke 方案要复杂得 多, 如考虑垂直速度, 地面感热通量、潜热通量等, 模式由于分辨率的粗略、物理过程的简化使得对这些 物理量的模拟能力较弱, 从而影响了 Tiedtke 方案的 性能. 另外, 敏感性试验结果表明不匹配的层状降水 方案对 Tiedtke 方案性能也有影响. 为从根本上解决 不匹配的层状降水方案问题, 下一步计划移植与 Tiedtke 方案匹配的显示云微物理方案, 以弥补模式
缺陷. 另外由于谱模式在用谱分量合成湿度场时会 出现下冲或上溢现象, 容易出现负水汽或过饱和的 情况, 从而直接影响模式的湿过程, 而采用半拉格朗 日格式并配合相应的保形插值方法可以避免上述湿 度计算问题, 这也是模式今后的改进方向.

上述研究表明, 引进 Tiedtk 方案后的 SAMIL 模 式在降水模拟上还存在一定问题, 特别是对层状降 水的模拟. 本文同时提出 Tiedtke 方案模拟偏差的可 能原因, 即与不匹配的层状降水方案有关, 需要引入 与之匹配的层状降水方案以此来改进模式.

致谢衰心感谢两位审稿专家对本文提出的建设性意见.

\section{参考文献}

1 Smagorinsky J. On the inclusion of moist-adiabatic processes in numerical prediction models. Beitr Dtsch Wetterdien, 1956, 5: 82-90

2 Manabe S, Smagorinsky J, Strickler R. Simulated climatology of a general circulation model with a hydrologic cycle. Mon Weather Rev, 1965, 93: 769-798

3 Kuo H L. On formation and intensification of tropical cyclones through latent heat release by cumulus convection. J Atmos Sci, 1965, 22: 40-63

4 Arakawa A, Schubert W H. Interaction of a cumulus cloud ensemble with the large-scale environment: Part I. J Atmos Sci, 1974, 31: 674-701

5 Tiedtke M. A comprehensive mass flux scheme for cumulus parameterization in large-scale models. Mon Weather Rev, 1989, 117: 1779-1800

6 王建捷, 周斌, 郭肖容. 不同对流参数化方案试验中凝结加热的特征及对暴雨中尺度模拟结果的影响. 气象学报, 2005, 63: 405-417

7 成安宁, 陈文, 黄荣辉. 积云对流参数化方案对气候数值模拟的影响. 大气科学, 1998, 22:814-824

8 曹杰, 张秀年, 尤亚否, 等. 区域气候模式(RegCM3)中积云对流参数化方案在纵向岭谷区的适用性研究. 科学通报, 2007, 52(增刊 II ): 101-106

9 Liu Y M, Guo L, Wu G X, et al. Sensitivity of ITCZ configuration to cumulus convective parameterizations on an aqua planet. Clim Dyn, 2010, 34: 223-240

10 Neale R B, Richter J H, Jochum M. The impact of convection on ENSO: From a delayed oscillator to a series of events. J Clim, 2008, 21: 5904-5924

11 贾小龙, 李崇银. 热带大气季节内振荡数值模拟对积云对流参数化方案的敏感性. 气象学报, 2007, 65: 837-855

12 李剑东, 刘屹岷, 孙治安, 等. 辐射和积云对流过程对大气辐射通量的影响. 气象学报, 2009, 67: 355-369

13 Arteta J, Marécal V, Rivière E D. Regional modeling of tracer transport by tropical convection. Part 1: Sensitivity to convection parameterization. Atmos Chem Phys, 2009, 9: 7081-7100

14 Lin J, Mapes B, Zhang M, et al. Stratiform precipitation, vertical heating profiles, and the Madden-Julian Oscillation. J Atmos Sci, 2004, 61: 296-309

15 吴国雄, 张学洪, 刘辉, 等. LASG 全球海洋-大气-陆面系统模式(GOALS/LASG)及其模拟研究. 应用气象学报, 1997, 8 (增刊): 15-28

16 王在志, 吴国雄, 刘平, 等. 全球海陆气耦合模式大气模式分量的发展及其气候模拟性能 I: 水平分辨率的影响. 热带气象学报, 2005, 21: 225-237

17 王在志, 宇如聪, 王鹏飞, 等. 全球海陆气耦合模式大气模式分量的发展及其气候模拟性能 II: 垂直分辨率的提高及其影响. 热带气 象学报, 2005, 21: 238-247

18 Wu T W, Liu P, Wang Z, et al. The performance of atmospheric component model R42L9 of GOALS/LASG. Adv Atmos Sci, 2003, 20: 726-742

19 吴统文, 吴国雄, 王在志, 等. GOALS/LASG 模式对气候平均态的模拟. 气象学报, 2004, 62: 20-31

20 宋晓良. 两种质量通量型积云参数化方案在气候模拟中的评估分析研究. 博士学位论文. 北京: 中国科学院研究生院, 2005 . 
$119-145$

21 刘屹岷, 刘琨, 吴国雄. 积云对流参数化方案对大气含水量及降水的影响. 大气科学, 2007, 31: 1201-1211

22 Edwards J M, Slingo A. Studies with a flexible new radiation code. I: Choosing a configuration for a large-scale model. Q J R Meteorol Soc, 1996, 122: 689-720

23 Slingo J M. A cloud parameterization scheme derived from GATE data for use with a numerical model. Q J R Meteorol Soc, 1980, 106: $747-770$

24 Slingo J M. The development and verification of a cloud prediction scheme for the ECMWF model. Q J R Meteorol Soc, 1987, 113: 899-927

25 戴福山. 东太平洋低云对海气耦合模式中“双辐合带”的影响——基于 LASG FGCM-0 的分析研究. 博士学位论文. 北京: 中国科学 院研究生院, 2003. 102-118

26 包庆, 刘屹峮, 周天军, 等. LASG/IAP 大气环流谱模式对陆面过程的敏感性试验. 大气科学, 2006, 30: 1077-1090

27 周天军, 宇如聪, 王在志, 等. 大气环流模式 SAMIL 及其耦合模式 FGOALS2s. 北京: 气象出版社, 2005. 288

28 Nordeng T E. Extended versions of the convective parameterization scheme at ECMWF and their impact on the mean and transient activity of the model in the tropics. Technical Memorandum, 1994

29 Adler R F, Susskind J, Huffman G J, et al. The Version-2 Global Precipitation Climatology Project (GPCP) monthly precipitation analysis (1979-Present). J Hydrometeorol, 2003, 4: 1147-1167

30 Xie P P, Arkin P A. Global precipitation: A 17-year monthly analysis based on gauge observations, satellite estimates and numerical model outputs. Bull Amer Meteorol Soc, 1997, 78: 2539-2558

31 Uppala S M, Kallberg P W, Simmons A J, et al. The ERA-40 re-analysis. Q J R Meteorol Soc, 2005, 131: 2961-3012

32 Kalnay E, Kanamitsu M, Kistler R, et al. The NCEP/ NCAR 40-year reanalysis project. Bull Amer Meteorol Soc, 1996, 77: 437-471

33 王在志, 宇如聪, 包庆, 等. 大气环流模式(SAMIL)海气耦合前后性能的比较. 大气科学, 2007, 31: 202-213

34 Houze R A. Observed structure of mesoscale convective systems and implications for large-scale heating. Q J R Meteorol Soc, 1989, 115: 425-461

35 Houze R A. Stratiform precipitation in regions of convection: A meteorological paradox? Bull Amer Meteorol Soc, 1997, 78: 2179-2196

36 Leary C A, Houze R A. The structure and evolution of convection in a tropical cloud cluster. J Atmos Sci, 1979, 36: 437-457

37 Cheng C P, Houze R A. The distribution of convective and mesoscale precipitation in GATE radar echo patterns. Mon Weather Rev, 1979, 107: 1370-1381

38 Roeckner E, Bauml G, Bonaventura L, et al. Part I: Model description. The Atmospheric General Circulation Model ECHAM5, 2004

39 刘屹岷, 吴国雄, 刘辉, 等. 空间非均匀加热对副热带高压形成和变异的影响III: 凝结潜热加热与南亚高压及西太平洋副高. 气象 学报, 1999, 57: 525-538

40 李崇银. 大气低频振荡. 北京: 气象出版社, 1991. 15-16

41 Taylor K E. Summarizing multiple aspect s of model performance in a single diagram. J Geophys Res, 2001, 106: 7183-7192 